\title{
Eficácia de mobiliário escolar adaptado de baixo custo no desempenho funcional de criança com paralisia cerebral ${ }^{1}$
}

\author{
Marco Aurélio Teixeira Piovezanni * \\ Aila Narene Dahwache Criado Rocha ** \\ Lígia Maria Presumido Braccialli ***
}

\section{Resumo}

Indivíduos com Paralisia Cerebral apresentam desenvolvimento motor atípico, caracterizado por alterações posturais, de coordenação motora e de tônus muscular, que resulta em limitações no desempenho de atividades funcionais. Neste contexto, destaca-se a prescrição e confecção de recursos de tecnologia assistiva com objetivo de maximizar as habilidades funcionais destas pessoas e prover sua inclusão social. Os indivíduos com paralisia cerebral, muitas vezes, têm dificuldade para a manutenção da dinâmica corporal, principalmente na postura sentada. O objetivo deste estudo foi o de confeccionar uma cadeira e mesa escolar adaptadas com material de baixo custo e verificar sua eficácia no desempenho grafomotor de uma criança com paralisia cerebral. O participante foi uma criança com diagnóstico de paralisia cerebral diplégica, do gênero masculino. A coleta e registro dos dados foram feitos em três momentos, com a criança posicionada no mobiliário adaptado, no mobiliário escolar comum e, novamente, no mobiliário adaptado, para se estabelecer a comparação. A análise de dados se deu por meio de testes estatísticos não paramétricos. Não houve significância estatística e foi verificada uma inconstância nos dados apresentados, já que não se pode afirmar ao certo qual mobiliário foi mais eficaz na realização da atividade proposta e nem se houve aprendizado motor com a sua repetição. Esse fato não invalida a adequação de mobiliário escolar ao aluno com paralisia cerebral, visto que este é um fator importante para favorecer o controle e estabilidade postural ao individuo, o que interfere na coordenação motora fina destes indivíduos, influenciando no seu desempenho em atividades escolares.

Palavras-chave: Educação Especial; Tecnologia assistiva; Mobiliário escolar.

\footnotetext{
* Terapeuta Ocupacional da Associação Beneficente de Assis. Graduado no curso de Terapia Ocupacional da UNESP - Marília. Assis, São Paulo, Brasil.

** Professora Doutora da Universidade Estadual Paulista Júlio de Mesquita Filho (UNESP) - Marília. Marília, São Paulo, Brasil.

** Professora Doutora da Universidade Estadual Paulista Júlio de Mesquita Filho (UNESP) - Marília. Marília, São Paulo, Brasil.
} 


\section{Effectiveness of low cost adapted school furniture on the functional performance of a child with cerebral palsy}

\section{Abstract}

Individuals with cerebral palsy present an atypical motor function, characteristic altered postures in movement coordination and muscle tone. This causes limitations in their ability to perform functional activities. In this context, the introduction of assistive technology is vital to the objective of augmenting their ability to function productively and be included in society. It is common for individuals with cerebral palsy to have difficulty maintaining body dynamics, especially with seated posture. The objective of this study is to produce an adapted school desk and adapted school chair with low cost materials and to study their efficacy in adjusting the writing motor skills of a child with cerebral palsy. This stydi's case is a boy who has been diagnosed with diplegic cerebral palsy. The collection and registration of data was done in three stages, with the child positioned in adapted furniture, regular school furniture and again in the furniture adapted to establish a comparison. Data analysis was through nonparametrical statistical tests. There was no statistical significance and was verified inconsistency in the data presented, because cannot be said for sure wich furniture was more effective in carrying while performing a proposed task and even if there was learning motor with its repetition. This fact does not invalidate the adequacy of school furniture to the student with cerebral palsy, because is an important factor facilitate control and postural stability to the individual, which interferes with fine motor skills of these individuals, influencing their performance in school activities.

Keywords: Special Education; Assistive Technology; School furniture.

\section{Introdução}

A Educação Especial deve garantir serviços de apoio especializados aos alunos com deficiência, transtornos globais do desenvolvimento e altas habilidades ou superdotação com o intuito de eliminar barreiras que limitem o processo de escolarização. Estes serviços constituem atendimentos que são identificados sob a denominação de Atendimento Educacional Especializado (AEE), conforme Decreto 7611 (BRASIL, 2011). Nas salas de recursos multifuncionais, que são dotadas de equipamentos, mobiliários e materiais didáticos e pedagógicos, para oferta deste apoio especializado, os objetivos do AEE são:

[...] Prover condições de acesso, participação e aprendizagem no ensino regular e garantir serviços de apoio especializados de acordo com as necessidades individuais dos estudantes; garantir a transversalidade das ações da educação especial no ensino regular; fomentar o desenvolvimento de recursos didáticos e pedagógicos que eliminem as barreiras no processo de ensino e 
aprendizagem; assegurar condições para a continuidade de estudos nos demais níveis, etapas e modalidades de ensino. (BRASIL, 2011, p. 12)

Dentre as limitações físicas, a Paralisia Cerebral (PC) é mais frequentemente encontrada, e o professor deste aluno, geralmente, necessita de diferentes dispositivos para facilitar e propiciar o seu ensino. Segundo o Consenso Internacional de 2005, Paralisia Cerebral pode ser definida como um grupo de desordens do desenvolvimento do movimento e da postura que causam limitação no desempenho de atividades, devido à distúrbios internos não progressivos que ocorreram no desenvolvimento fetal ou infantil do cérebro. Os distúrbios motores da paralisia cerebral são frequentemente acompanhados por alterações da cognição, sensação, comunicação, percepção e/ou comportamento, e/ou por uma dificuldade de aprendizado (BAX et al., 2005).

Uma das principais dificuldades encontradas pelos alunos com deficiência física, no contexto escolar, é a acessibilidade do espaço (MACHADO, 2007). A autora, ainda, ressalta que a maioria dos edifícios escolares é construído sem considerar as necessidades das pessoas com deficiência. Este fato limita a participação de alunos com deficiência física nas atividades escolares. A acessibilidade deve ser assegurada por meio da eliminação de barreiras arquitetônicas, urbanísticas, nas instalações, equipamentos e mobiliário, no transporte, além da eliminação das barreiras de comunicação e de informação (BRASIL, 2008).

Nessa perspectiva, a proposta de atendimento educacional especializado para alunos com deficiência física deve considerar as atitudes que permeiam o ambiente escolar e também as dimensões físicas do ambiente, que vão desde as condições de acesso, como a adequação do mobiliário escolar, o transporte adaptado e a arquitetura dos prédios escolares, até as barreiras discriminatórias que limitam a permanência com sucesso na escola (ALVES, 2006).

As ações direcionadas para a acessibilidade do aluno com deficiência abrangem conhecimento amplo e complexo no âmbito da tecnologia assistiva, que pode ser definida como:

[...] uma área do conhecimento, de característica interdisciplinar, que engloba produtos, recursos, metodologias, estratégias, práticas e serviços que objetivam promover a funcionalidade, relacionada à atividade e participação de pessoas com deficiência, incapacidades ou mobilidade reduzida, visando sua autonomia, independência, qualidade de vida e inclusão social. (CAT, 2007)

Segundo Braccialli (2007), recursos de tecnologia assistiva podem ser classificados em recursos de alta e baixa tecnologia. Este estudo terá como foco os recursos de baixa tecnologia, que podem ser definidos como, recursos com pouca sofisticação, confeccionados com material de baixo custo, por profissionais de saúde e educação e pelos próprios familiares. A autora ainda ressalta o predomínio destes recursos no país, fato provavelmente relacionado com o perfil socioeconômico da maior parte da população.

Revista Educação Especial | v. 27 | n. 49 | p. 485-498| maio/ago. 2014

Santa Maria 
O acesso de pessoas com deficiência a recursos de alta tecnologia está garantido por lei, como mostra o decreto 6949 de 2009, da Convenção Internacional sobre os Direitos da Pessoa com Deficiência. Cabe ao Estado assegurar que essas pessoas tenham acesso, em igualdade de oportunidades, como as demais pessoas, ao meio físico, transporte, informação e comunicação, em diferentes contextos, inclusive o escolar. Para isso, o mesmo deve possibilitar o acesso à recursos tecnológicos e a assistência apropriada, sendo necessária, muitas vezes, modificações no ambiente (BRASIL, 2009). Apesar de terem esse direito garantido por lei, o que, muitas vezes, observa-se é a falta de investimento público para a criação de novas tecnologias, que associado à falta de material e de recursos disponíveis, comprovam a importância e o predomínio das adaptações de baixo custo no país. (HOHMANN, CASSAPIAN, 2011).

Existe uma classificação para os recursos de tecnologia assistiva, no espaço escolar: (a) recursos para Comunicação alternativa; (b) adequação de materiais pedagógicos; (c) adequação de recursos de informática; (d) projetos para acessibilidade arquitetônica e (e) adequação de mobiliário (BERSCH, MACHADO, 2007). A ausência de adaptações do espaço físico, mobiliário escolar adaptado e demais recursos de tecnologia assistiva implicam em um prejuízo funcional para o individuo, comprometendo sua qualidade de vida (ALPINO 2008).

Indivíduos com paralisia cerebral, em sua maioria, têm dificuldade para a manutenção da dinâmica corporal, principalmente na postura sentada, na qual é desenvolvida a maioria das atividades escolares, portanto se faz necessário pensar em recursos que possibilitem um sentar adequado, que ofereça conforto e segurança (WASHINGTON et al., 2002). O alinhamento e a estabilidade postural são questões fundamentais para a realização de qualquer atividade, inclusive as escolares. Uma postura adequada vai trazer vários benefícios para aluno em sala de aula, dentre eles, facilidade para manter o contato visual, para realizar atividades de manipulação com os membros superiores, o menor gasto energético e maior atenção durante a realização de atividades escolares (BERSCH, 2007). A autora ainda ressalta que, em termos motores, o alinhamento postural promove a melhora do tônus muscular e a diminuição de movimentos involuntários, o que permite uma maior coordenação de movimentos para a realização de atividades.

É importante ressaltar que não existe uma cadeira ideal para todos os indivíduos, porque é necessário considerar as necessidades de cada um, de acordo com suas medidas antropométricas (OLIVEIRA, 2007; SARAIVA e MELO, 2011). O tipo de assento, a regulação de sua inclinação e de seu encosto e as características da mesa de apoio são aspectos que devem ser considerados para proporcionar uma postura adequada e um maior conforto ao indivíduo (OLIVEIRA, 2007).

Segundo Braccialli (2000), deve-se ter cautela na prescrição e confecção de um mobiliário adaptado, visto que uma cadeira mal projetada pode causar: dormência e formigamento nos membros, dificuldades no retorno venoso, dores lombares, aceleração do processo degenerativo do disco intervertebral, desgaste das vértebras e, consequentemente, fadiga muscular durante a realização de atividades. 
Nesse contexto, Saraiva e Melo (2011) e Alpino (2008), ressaltam a importância do trabalho de profissionais da área de saúde, como o fisioterapeuta e o terapeuta ocupacional, que devem, principalmente, trabalhar junto com o professor com as questões posturais, de locomoção e de adequação de mobiliário. A presença destes profissionais está garantida pela Lei de Diretrizes e Bases da Educação (lei 9394/96), que estabelece que: na modalidade de Educação Especial, haverá, quando necessário, a presença de serviços de apoio especializado para atender as especificidades dos educandos (BRASIL, 1996). É importante ressaltar que estes profissionais devem avaliar, de forma contínua, os alunos em seu mobiliário, para buscar um melhor posicionamento e funcionalidade em sala de aula, promovendo uma maior resposta de ensino-aprendizagem, além de realizar consultoria e orientação aos pais e demais profissionais da equipe (GIACOMINI, SARTORETTO, BERSCH, 2010).

Em relação à adequação do mobiliário escolar a alunos com deficiência física, a literatura aponta muitas dificuldades neste processo, como, mobiliário que não atende às necessidades específicas de posicionamento do aluno; ausência de profissional capacitado para prescrevê-lo; estado de conservação precário do mobiliário; falta de informação da equipe escolar; e dificuldades de comunicação com a Secretaria da Educação (SARAIVA e MELO, 2011). Todos estes fatores contribuem para uma dificuldade de permanência do aluno com paralisia cerebral no ensino regular.

Em um estudo realizado por Alpino (2003), os professores tiveram a percepção da importância de apoio especializado para inclusão de alunos com deficiência no ensino regular, relatando ser necessária a adequação do mobiliário escolar, adaptações do espaço físico, dos recursos e dos materiais pedagógicos.

Com base nas informações anteriores, percebe-se a necessidade de prescrever e confeccionar mobiliários e recursos de tecnologia assistiva de baixo custo com qualidade, para que sejam mais acessíveis à sociedade e permitam um melhor posicionamento, função e qualidade de vida para os alunos que necessitam destes dispositivos. E nessa direção, o objetivo do presente estudo é verificar a eficácia de uma cadeira e mesa escolar adaptadas confeccionadas com material de baixo custo no desempenho grafomotor de uma criança com paralisia cerebral.

\section{Método}

\section{Procedimentos éticos}

O projeto foi aprovado pelo Comitê de Ética em Pesquisa da Faculdade de Filosofia e Ciências, Unesp - Marília, sob o no 0225/201l. O projeto também foi cadastrado no Centro de Estudos da Educação e da Saúde (CEES) e no Laboratório de Desempenho Motor (LADEMO) da Faculdade de Filosofia e Ciências, Unesp - Marília. A realização da pesquisa ocorreu conforme orienta a Resolução 196/96, no que concerne à concordância do participante/responsável com o Termo de Consentimento Livre e Esclarecido. 


\section{Participante}

Adotaram-se como critérios de inclusão do participante: ter diagnóstico de paralisia cerebral, não apresentar déficit cognitivo acentuado, possuir uma boa função em pelo menos um dos membros superiores, estar em idade escolar e não possuir mobiliário adaptado na escola. Foi selecionada, para o estudo, uma criança com diagnóstico de paralisia cerebral, do tipo diplegia espástica, do gênero masculino, com seis anos de idade, nível III no Sistema de Classificação da Função Motora Grossa (GMFCS) e nível II no Sistema de Classificação da Habilidade Manual (MACS). A criança é atendida nos setores de fonoaudiologia e fisioterapia, no CEES, da Faculdade de Filosofia e Ciências da Unesp - campus de Marília. O participante frequenta uma Escola Municipal de Educação Infantil (EMEI), no período da tarde, mas não possui um mobiliário adaptado, e, durante o período escolar, fica posicionado em uma cadeira de rodas.

\section{Local}

O estudo foi desenvolvido no LADEMO da Faculdade de Filosofia e Ciências Unesp - campus de Marília.

\section{Materiais e equipamentos}

Foram utilizados os seguintes materiais para a confecção do mobiliário adaptado: 100 garrafas PoliTereftalato de Etileno ( PET) de 2 litros que foram obtidas por meio de doações, tesoura, fita adesiva larga, espuma caixa de ovo, papelão, cola quente, abraçadeira de nylon ("enforca-gato"), tecido para revestimento, feltro, velcro, caixa de papelão, EVA, flutuador espaguete de Polietileno ("espaguete de piscina"), manta acrílica e cola de contato.

Os equipamentos usados durante a coleta de dados foram: a cadeira adaptada e mesa recortada confeccionadas, mesa e cadeira escolar comum, computador e mesa digitalizadora da marca Wacom - Intuos 3. Para análise da produção gráfica do sujeito da pesquisa foi usado o software MovAlyseR 6.l. A Figura 1 ilustra a mesa digitalizadora utilizada para coletar os dados do desempenho grafomotor do participante.

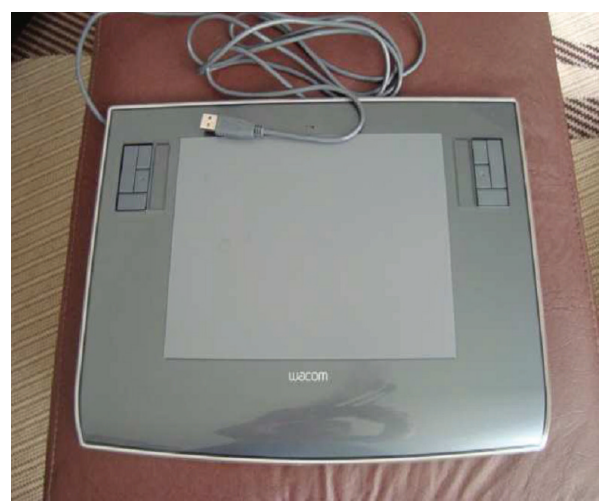

Figura 1 - A mesa digitalizadora utilizada na coleta de dados. Fonte: CODOGNO (2011). 


\section{Confecção do mobiliário adaptado}

Para a confecção do mobiliário adaptado, composto por conjunto mesa e cadeira, foi realizado um projeto de um recurso que apresentasse as seguintes características: conforto, segurança, resistência e funcionalidade. A cadeira foi confeccionada seguindo a técnica do Professor Sebastião Feijó para confecção de cadeiras com garrafa PET, com algumas modificações (RECICLOTECA, [20--]). A mesa, com recorte, foi feita com papelão e velcro para fixá-la na cadeira, e foi revestida com papel EVA. Foram confeccionadas as seguintes adaptações adicionais para adequação postural do usuário, tendo em vista suas características motoras: abdutor, apoio pélvico lateral, cinto pélvico e apoio de pé (VANZETTI, 2009). Todas as adaptações foram confeccionadas com material de baixo custo.

Para confeccionar o mobiliário, foram realizadas as seguintes medidas antropométricas do participante: a) comprimento do segmento da perna do aluno, da prega poplítea até a planta do pé $(33 \mathrm{~cm})$; b) Comprimento do fêmur partindo da prega poplítea até a borda dos glúteos $(33 \mathrm{~cm})$; e c) distância entre os quadris $(26 \mathrm{~cm})$. A Figura 2 ilustra a cadeira adaptada confeccionada. A Figura 3 apresenta a mesa recortada confeccionada para a coleta de dados.

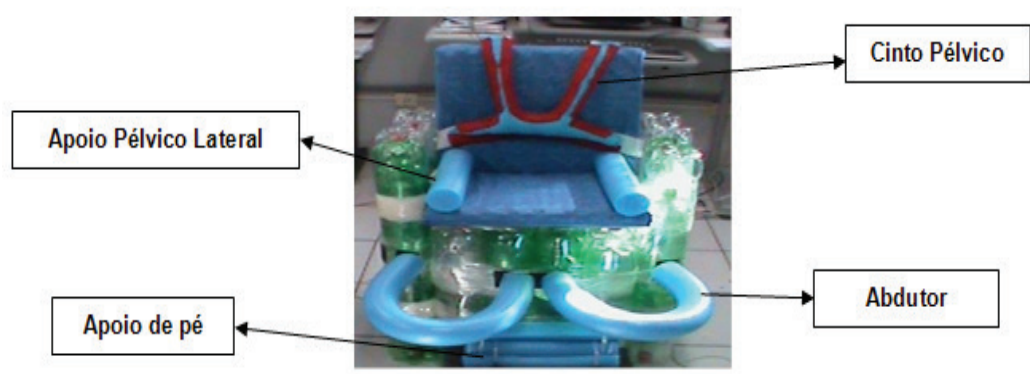

Figura 2 - Cadeira confeccionada, em fase de finalização, com as adaptações necessárias ao participante para a coleta de dados. Fonte: Produção própria.

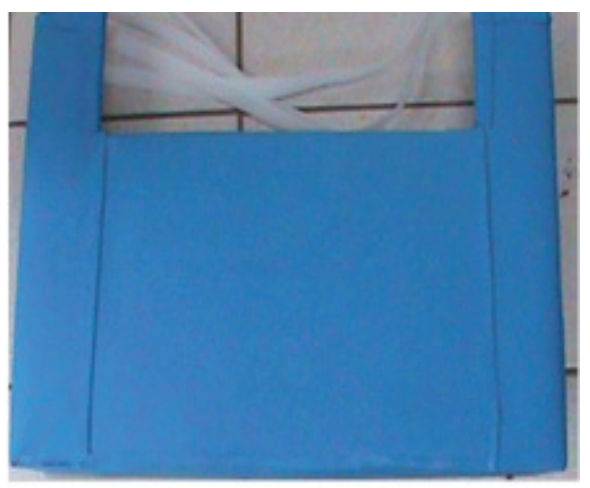

Figura 3 - Mesa recortada utilizada na coleta de dados.

Fonte: Produção própria.

Revista Educação Especial | v. 27 | n. 49 | p. 485-498| maio/ago. 2014

Santa Maria

Disponível em: <http://www.ufsm.br/revistaeducacaoespecial> 
Durante a coleta de dados, foi notada que a altura do encosto e a área de contato do assento do mobiliário adaptado estavam adequadas. Pode-se observar, também, que os materiais utilizados para a confecção da cadeira, proporcionaram, juntamente com os demais recursos para adequação postural confeccionados, uma maior durabilidade e resistência do mobiliário, além de proporcionar uma maior estabilidade ao usuário, tendo em vista suas características motoras.

\section{Coleta de dados}

Previamente, foi realizado o treinamento do pesquisador para a utilização do equipamento. Durante a coleta a criança foi posicionada sentada na cadeira adaptada e na cadeira escolar comum, de costas para a tela do computador, para não perder a atenção durante a atividade grafomotora proposta. Sobre a mesa adaptada, foi posicionada a mesa digitalizadora que continha uma folha de sulfite A4, na qual foi realizada a atividade, que consistiu em realizar o tracejado de um desenho. A criança utilizou a caneta digital (Ink Pen) do equipamento. A mesa digitalizadora estava conectada via USB em um computador que continha o respectivo software para a coleta e análise dos dados. A Figura 4 ilustra a atividade grafomotora realizada na coleta de dados.

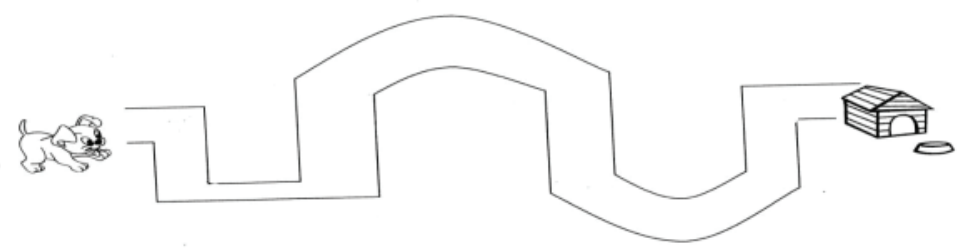

Figura 4 - Atividade grafomotora proposta para a coleta de dados.

Fonte: ATIVIDADES (2011).

A coleta de dados foi feita com ajuda de uma auxiliar de pesquisa e durou cerca de 60 minutos. Esse procedimento teve três momentos: a) no primeiro, a criança foi posicionada no mobiliário adaptado; b) no segundo momento, a criança foi posicionada no mobiliário escolar comum; c) no terceiro e último momento, a criança foi posicionada, novamente, no mobiliário adaptado. A atividade foi realizada dez vezes em cada momento. A Figura 5 ilustra o participante posicionado em dois momentos diferentes da coleta de dados.

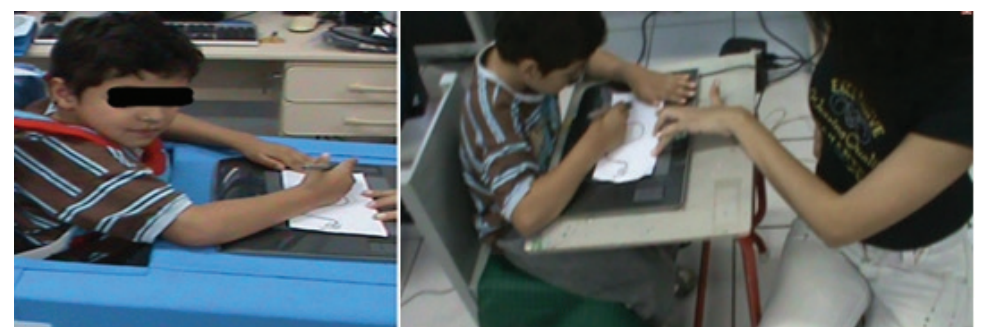

Figura 5 - À esquerda, participante posicionado no mobiliário adaptado e à direita posicionado no mobiliário escolar comum, durante a coleta de dados.

Fonte: Produção própria. 


\section{Análise dos dados}

O desempenho grafomotor do participante foi avaliado com o software MovAlyseR 6.1, por meio das seguintes variáveis: tempo de duração da atividade, velocidade (capacidade de deslocar a caneta no espaço-tempo), aceleração (capacidade do indivíduo para alterar sua aceleração durante o movimento) e pressão da caneta no papel.

Para a análise dos dados de tempo de duração da atividade, velocidade, aceleração e pressão da caneta foi feito o teste de normalidade de Shapiro Wilk. O resultado do teste de normalidade de Shapiro Wilk foi significante $(\mathrm{p}<0,05)$ para as variáveis em estudo, motivo pelo qual as análises estatísticas entre os momentos: mobiliário adaptado (MA), mobiliário comum (MC) e mobiliário adaptadol (MAl) foram realizadas por meio da análise de variância não paramétrica de Friedman (medidas repetidas). Adotou-se, para todos os testes, o nível de significância de 5\% de probabilidade para a rejeição da hipótese de nulidade $(\mathrm{p} \leq 0.05)$.

\section{Resultados}

Na Tabela 1, é apresentada a análise dos dados da variável tempo de duração da atividade. Observa-se que não há diferença significante entre os três momentos de avaliação, com o uso do mobiliário adaptado e mobiliário escolar comum.

Tabela 1 - Análise estatística não paramétrica da variável Tempo de duração da atividade.

\begin{tabular}{ccccc}
\hline \multicolumn{5}{c}{ Tempo de duração da atividade (s) } \\
\hline Média & Desvio Padrão & Mínimo & Máximo \\
\hline MA & 0,908 & 0,239 & 0,657 & 1,316 \\
\hline MC & 0,853 & 0,422 & 0,464 & 1,833 \\
\hline MAl & 0,852 & 0,482 & 0,332 & 1,865 \\
\hline
\end{tabular}

Na Tabela 2, é apresentada a análise dos dados da variável velocidade, sendo que também não houve diferença significante nos três momentos da coleta de dados, com o mobiliário adaptado e com o mobiliário escolar comum.

Tabela 2 - Análise estatística não paramétrica da variável velocidade.

\begin{tabular}{ccccc}
\hline & \multicolumn{5}{c}{ Velocidade (m/s) } \\
\hline Média & Desvio Padrão & Mínimo & Máximo \\
\hline MC & 3,878 & 1,381 & 1,72 & 6,428 \\
\hline MAl & 4,813 & 1,858 & 3,402 & 9,418 \\
\hline & 4,685 & 1,715 & 1,927 & 7,256 \\
\hline
\end{tabular}

Na Tabela 3, encontra-se a análise dos dados da variável aceleração. Não houve diferença significante nos três momentos da coleta de dados, com os dois mobiliários utilizados na pesquisa. 
Marco Aurélio Teixeira Piovezanni - Aila Narene Dahwache Criado Rocha - Lígia Maria

Presumido Braccialli

Tabela 3 - Análise estatística não paramétrica da variável aceleração.

\begin{tabular}{ccccc}
\hline \multicolumn{5}{c}{ Aceleração (cm/s2) } \\
\hline Média & Desvio Padrão & Mínimo & Máximo \\
\hline MA & 40,174 & 57,880 & $-10,836$ & 183,027 \\
\hline MC & 72,876 & 107,387 & 10,971 & 348,081 \\
\hline MAl & 36,735 & 6,015 & $-0,088$ & 106,882 \\
\hline
\end{tabular}

Na Tabela 4, é apresentada a análise dos dados da variável pressão da caneta. É possível notar que não houve diferença significante nos três momentos de avaliação, com a utilização do mobiliário adaptado e do mobiliário escolar comum.

Tabela 4 - Análise estatística não paramétrica da variável pressão da caneta.

\begin{tabular}{ccccc}
\hline \multicolumn{5}{c}{ Pressão na caneta (N) } \\
& Média & Desvio Padrão & Mínimo & Máximo \\
\hline MA & 581,305 & 82,220 & 454.031 & 700,867 \\
\hline MC & 587,864 & 270,080 & 23.549 & 877,585 \\
\hline MAl & 681,715 & 123,722 & 470.194 & 866,192 \\
\hline & $\mathrm{p}=0.882$
\end{tabular}

\section{Discussão}

Na Tabela l, pode-se observar que na variável tempo de duração da atividade, houve uma diminuição gradativa dos valores, o melhor desempenho do participante foi no $3^{0}$ momento, no mobiliário adaptado, já que nesse momento a criança concluiu a atividade de forma mais rápida (média=0,852s).

A análise da Tabela 2 indica que a variável velocidade aumentou no $2^{\circ}$ e $3^{\circ}$ momentos de avaliação; entretanto, o melhor desempenho foi no $2^{\circ}$ momento, no mobiliário escolar comum, já que foi nessa etapa em que o participante concluiu a atividade de forma mais rápida (média=4,813m/s).

A Tabela 3 mostra que houve um aumento considerável na aceleração no $2^{\circ}$ momento, porém no $3^{\circ}$ momento esse valor diminuiu, e nesse caso o melhor desempenho do participante foi no mobiliário escolar comum (média=72,876 cm/s2).

Esses resultados ocorreram, provavelmente, devido ao fato da atividade ser repetida dez vezes em cada mobiliário, o que permitiu a aprendizagem do sujeito, e contribui para um melhor desempenho do participante no $2^{\underline{0}}$ e $3^{\circ}$ momentos da coleta de dados, e sendo assim, nessas variáveis não houve tanta interferência do tipo de mobiliário utilizado, esse mesmo fato ocorreu no estudo de Codogno (2011). A autora ainda ressalta que esse acontecimento não invalida a necessidade de adequação do mobiliário escolar, fator importante para o controle postural e para o desempenho de atividades que requerem coordenação motora fina. Calvo (2007), ainda pontua que a prática motora e a aprendizagem também interferem na coordenação motora fina de alunos com comprometimento motor. 
Na Tabela 4, observa-se que a variável pressão da caneta teve um aumento gradual no $2^{\underline{o}}$ e $3^{\underline{0}}$ momentos de avaliação, portanto o maior valor foi no mobiliário adaptado no $3^{\circ}$ momento (média=681,715N). No mobiliário escolar comum, a pressão da caneta chegou a $877,585 \mathrm{~N}$, que foi o maior valor encontrado. Nesse caso, nem sempre o maior valor indica um melhor desempenho do participante, visto que a criança com paralisia cerebral apresenta um déficit no controle motor e controle postural, o que influencia em sua coordenação motora fina, esse valor pode indicar, ainda, um desgaste físico do aluno durante a realização da atividade (CODOGNO, 2011). Portanto pode-se notar que de alguma forma o mobiliário adaptado interferiu nessa variável, visto que a maior pressão sobre a caneta ocorreu no mobiliário escolar comum, sendo que este, provavelmente, não favorece um alinhamento e estabilidade postural adequados, além da melhor coordenação motora de membros superiores e menor gasto energético, que são características de um mobiliário adaptado que atende às necessidades do aluno com paralisia cerebral, como preconiza Bersch (2007).

Além dos dados das variáveis estudadas não apresentarem significância estatística, foi verificada uma inconstância nos mesmos, já que não se pode afirmar qual mobiliário foi mais eficaz, visto que o participante teve um melhor desempenho nas variáveis pressão na caneta e tempo de duração no mobiliário adaptado e melhor desempenho nas variáveis velocidade e aceleração no mobiliário escolar comum, assim, não se pode afirmar com precisão se houve aprendizado motor, pois apenas na variável tempo de duração o desempenho foi melhor na última etapa de avaliação.

No estudo de Gonçalves (2008), foi utilizado um software para avaliação de habilidades motoras finas de indivíduos hemiparéticos, para tanto, foi proposta uma atividade grafomotora semelhante a do presente estudo, visto que a mesma tinha o objetivo de levar a caneta de uma mesa digitalizadora do ponto inicial até o ponto final de uma linha pré-estabelecida na tela. O autor não encontrou diferença estatística significante ao avaliar o desempenho dos participantes na atividade, e ressalta ainda que isso ocorreu devido ao comprometimento motor apresentado pelos sujeitos do estudo, sendo que em alguns casos foi necessário adaptar a caneta e alguns participantes apresentavam movimentos compensatórios. Portanto, pode-se dizer que o quadro motor influencia o desempenho em atividades grafomotoras, este pode ser um fator que, possivelmente, influenciou os resultados encontrados no presente estudo.

O estudo realizado por Monteiro, et al (2010), tinha como objetivo analisar o processo de aprendizagem motora de crianças com paralisia cerebral do tipo diplegia espástica em uma tarefa com o computador, na qual os participantes (grupo controle e grupo experimental) deveriam achar o caminho certo em um pequeno labirinto. Para analisar os dados os autores utilizaram o mesmo teste estatístico do presente estudo, o de Friedman (para medidas repetidas), porém diferentemente desta pesquisa, obtiveram uma significância estatística e comprovaram que com a repetição houve aprendizado motor e que os participantes obtiveram um melhor desempenho na atividade proposta, realizando-a em menos tempo. Porém, os participantes desta pesquisa tinham uma função motora melhor que o sujeito do presente estudo, visto que apresentavam nível II no GMFCS, além disso, a atividade foi repetida em um maior 
número de vezes, 45 vezes, em 4 momentos distintos (30 no primeiro momento e 5 em cada momento posterior), e, ainda, a atividade proposta possui uma demanda motora inferior a atividade proposta no presente estudo, pois foi realizada com o teclado do computador. Para os autores, um fator importante que influenciou o desempenho dos participantes do grupo controle, foi a motivação pela atividade apresentada pelos mesmos. Provavelmente esses fatores influenciaram os resultados do estudo e possibilitaram um melhor desempenho dos participantes com a repetição da atividade.

Contudo, é necessário destacar que existem fatores intrínsecos e extrínsecos que podem interferir no desempenho motor de crianças com paralisia cerebral. No caso deste estudo, dois fatores intrínsecos podem ter interferido no desempenho do participante: a motivação frente a realização da atividade e o quadro motor caracterizado por alterações de tônus muscular, na coordenação de movimentos e de controle postural. Os fatores extrínsecos, que podem ter influenciado no desempenho da criança foram: o clima, o local onde foi realizada a pesquisa, os procedimentos de coleta de dados, e a atividade proposta. Possivelmente, houve alguma interferência de algumas destas variáveis no estudo, e, provavelmente, por isso os resultados obtidos apresentaram inconstância. Ressalta-se a importância de se realizarem novos estudos que avaliem a coordenação motora fina de crianças com paralisia cerebral, e que auxiliem a encontrar estratégias para proporcionar o aprendizado destes alunos, que podem ser a adequação do mobiliário escolar, a adaptação de recursos pedagógicos, modificações no ambiente, uso de tecnologia apropriada, entre outros.

\section{Conclusão}

O mobiliário adaptado confeccionado especificamente para o participante do estudo não interferiu no desempenho grafomotor durante a atividade realizada. Mesmo assim, este fato não anula a importância da presença de um mobiliário escolar adequado às necessidades dos alunos, principalmente alunos com paralisia cerebral, já que este mobiliário irá proporcionar um melhor alinhamento e estabilidade postural, maior função de membros superiores, e melhor desempenho em atividades escolares.

Ressalta-se a importância de se realizar novos estudos que avaliem a influência do mobiliário na coordenação motora fina de crianças com paralisia cerebral. É importante, também, pontuar algumas dificuldades encontradas em estudo com esta população-alvo, já que há a interferência de fatores intrínsecos e extrínsecos, mesmo com a utilização de critérios rigorosos para seleção dos participantes.

\section{Referências}

ALPINO, A. M. S. O aluno com paralisia cerebral no ensino regular: ator ou expectador do processo educacional? 2003. 14lf. Dissertação (Mestrado em Educação Especial) - Universidade Federal de São Carlos, São Carlos, 2003.

Consultoria colaborativa escolar do fisioterapeuta: acessibilidade e participação do aluno com paralisia cerebral em questão. 2008. 19lf. Tese (Doutorado em Educação Especial) - Universidade Federal de São Carlos, São Carlos, 2008.

ALVES, D. de O. Sala de recursos multifuncionais: espaços para atendimento educacional especializado. Brasília, DF: Ministério da educação (MEC), Secretaria de educação especial (SEESP), 2006. 
ATIVIDADES de coordenação motora. In: Espaço Educar. 2011. Disponível em: 〈http://espacoeducar-liza. blogspot.com/2009/02/atividades-de-coordenacao-motora.html〉. Acesso em: 20 mai. 2012

BAX, M., et al. Proposed definition and classification of cerebral palsy. Developmental Medicine and Child Neurology, v. 47, n. 8, p. 571-576, Aug. 2005. Disponível em: 〈http://onlinelibrary.wiley.com/ doi/10.11l1/j.1469-8749.2005.tb01195.x/pdf . Acesso em: 5 ago. 2011.

BERSCH, R. Alinhamento e estabilidade postural: Colaborando com as questões de aprendizado. In: SCHIRMER, C. R. Atendimento educacional especializado: Deficiência física. SEESP/SEED/MEC. Brasília, 2007, p.111-125.

BERSCH, R.; MACHADO, R. Atendimento educacional especializado para deficiência física. In: SCHIR MER, C. R. Atendimento educacional especializado: Deficiência física. SEESP/SEED/MEC. Brasília, 2007, p. $27-28$.

BRACCIALLI, L. M. P. Influência da utilização do mobiliário adaptado na postura sentada de indivíduos com paralisia cerebral espástica. 2000. 118 f. Tese (Doutorado em Educação Física)-Faculdade de Educação Física, Universidade Estadual de Campinas, Campinas, 2000. Disponível em: 〈http://www. bibliotecadigital.unicamp.br/document/?down=vtls000220000 >. Acesso: 10 jul. 2011.

. Tecnologia assistiva: perspectiva de qualidade de vida para pessoas com deficiência. In: VILARTA, R. et al. (Org.). Qualidade de vida e novas tecnologias. Campinas: IPES, 2007. p. 105-114. Disponível em: 〈www.bibliotecadigital.unicamp.br/document/?down=000410337〉. Acesso em: 10 jul. 2011.

BRASIL. DECRETO №9394, de 20 de dezembro de 1996. Regulamenta a Lei no 7.853, de 24 de outubro de 1989, Estabelece as diretrizes e bases da educação nacional. Disponível em: ‘http://www.planalto.gov. br/ccivil_03/leis/L9394.htm>. Acesso em: 20 mai. 2012.

Política nacional de Educação Especial na Perspectiva da Educação Inclusiva. Inclusão: R. Educ. esp., Brasília: MEC/SEESP, v. 4, n. 1 p. 7-17, 2008.

. Decreto №6949, de 25 de agosto de 2009a. Promulga a Convenção Internacional sobre os Direitos das Pessoas com Deficiência e seu Protocolo Facultativo, assinados em Nova York, em 30 de março de 2007. Disponível em:〈http://www.planalto.gov.br/ccivil_03/_Ato2007-2010/2009/Decreto/D6949.htm〉. Acesso em: 15 jul. 2012.

Decreto №7611, de 17 de novembro de 2011. Dispõe sobre a educação especial, o atendimento educacional especializado e dá outras providências. Disponível em: 〈http://www.planalto.gov.br/ccivil_03/_ Ato2011-2014/2011/Decreto/D7611.htm\#artll’. Acesso em: 2 abr. 2012.

CALVO, A. P. A produção gráfica e a escrita: focalizando a variação da produção de força. 2007. 173 f. Dissertação (Mestrado em Ciência da Motricidade Humana) - Instituto de Biociências, Universidade Estadual Paulista, Rio Claro, 2007.

CODOGNO, F. T. O. Influência do mobiliário na coordenação motora fina e no controle postural de alunos com paralisia cerebral. 2001. 14lf. Tese (Doutorado em Educação), Faculdade de Filosofia e Ciências, Universidade Estadual Paulista, Marília, 2011.

COMITÊ DE AJUDAS TÉCNICAS (CAT). Secretaria Especial dos Direitos Humanos da Presidência da República (SEDH/PR). Ata VII Reunião do Comitê de Ajudas Técnicas - CAT. Brasília, DF, 2007. Disponível em: 〈http://portal.mj.gov.br/corde/arquivos/doc/Ata_VII_Reuni\%C3\%A3o_do_Comite_de_Ajudas_T\%C3\%A9cnicas.doc >. Acesso em: 20 nov. 2012.

GIACOMINI, L.; SARTORETTO, M. L.; BERSCH, R. C. R. A Educação especial na perspectiva da inclusão escolar: orientação e mobilidade, adequação postural e acessibilidade espacial. Brasília: MEC, Secretaria da Educação Especial, Universidade Federal do Ceará, 2010.

GONÇALVES, V. P. Software de aprendizagem e controle motor para avaliação de indivíduos hemiparéticos: validade e confiabilidade. 2008. 14lf. Dissertação (Mestrado em Ciências do Movimento Humano), Universidade do Estado de Santa Catarina, Florianópolis, 2008.

HOHMANN, P.; CASSAPIAN, M. R. Adaptações de baixo custo: uma revisão de literatura da utilização por terapeutas ocupacionais brasileiros. Revista Terapia Ocupacional. Univ. São Paulo. São Paulo, v. 22, n. 1, p. 10-18, 2011 .

MACHADO, R. Acessibilidade arquitetônica. In: SCHIRMER, C. R. Atendimento educacional especializado: Deficiência física. SEESP/SEED/MEC. Brasília, 2007, p. 105-108. 
MONTEIRO, C. B. M., et al. Aprendizagem motora em crianças com paralisia cerebral. Revista Brasileira Crescimento e Desenvolvimento Humano, São Paulo, v. 20, n. 2, p. 250-262, 2010.

OLIVEIRA F. T. Estudo do mobiliário escolar durante o desempenho de atividades lúdicas por alunos com paralisia cerebral espástica. 2007. 100 f. Dissertação (Mestrado em Educação), Faculdade de Filosofia e Ciências, Universidade Estadual Paulista, Marília, 2007.

RECICLOTECA - Centro de informações sobre reciclagem e meio ambiente. Cadeira com Garrafas PET. Rio de Janeiro, [20--]. Disponível em: 〈http://www.recicloteca.org.br/passo.asp?Ancora=3〉. Acesso em: 28 jul. 2011.

SARAIVA, L. L. O.; MELO, F. R. L. V. Avaliação e participação do fisioterapeuta na prescrição do mobiliário escolar utilizado por alunos com paralisia cerebral em escolas estaduais públicas da rede regular de ensino. Revista Brasileira de Educação Especial, Marília, v. 17, n. 2, p. 245-262, 2011.

VANZETTI. Medida do paciente: cadeira de rodas. São Paulo, 2009. Disponível em: 〈http://www.vanzetti.com.br/portal/index. php/categoria/medida-paciente/cadeira-rodas/>. Acesso em: 22 mai. 2012.

WASHINGTON, K., et al. The effects of a countoured foam seat on postural alignment and upper-extremity function in infants with neuromotor impairments. Physical Therapy, v. 82, n. 11, p. 1064-1076, 2002. Disponível em: 〈http://ptjournal.apta.org/content/82/11/1064.full.pdf 〉. Acesso em: 26 jul. 2011.

\section{Nota}

${ }^{1} \mathrm{O}$ presente estudo é um recorte do projeto de iniciação científica do primeiro autor.

\section{Correspondência}

Lígia Maria Presumido Braccialli - Departamento de Educação Especial, Av. Hygino Muzzi Filho, 737. Bairro: Câmpus Universitário. CEP:17.525-900 - Marília, São Paulo - Brasil.

E-mail:bracci@marilia.unesp.br - marco_piovezanni@yahoo.com.br - aila@marilia.unesp.br

Recebido em 19 de março de 2013

Aprovado em 17 de novembro de 2013 